\title{
Lifting cosmic degeneracy within a single quasar survey
}

\author{
B. F. Roukema ${ }^{1}$ and G. A. Mamon ${ }^{2,3}$ \\ 1 Inter-University Centre for Astronomy and Astrophysics, Post Bag 4, Ganeshkhind, Pune, 411 007, India \\ e-mail: boud@iucaa.ernet.in \\ 2 Institut d'Astrophysique de Paris (CNRS UPR 341), 98bis Bd Arago, 75014 Paris, France \\ e-mail: gam@iap.fr \\ 3 DAEC (CNRS UMR 8631), Observatoire de Paris-Meudon, 5 place Jules Janssen, 92195 Meudon Cedex, France
}

Received 8 September 2000 / Accepted 19 October 2000

\begin{abstract}
In the almost Friedmann-Lemaitre model of the Universe, the density parameter, $\Omega_{\mathrm{m}}$, and the cosmological constant, $\Omega_{\Lambda}$, measure curvature. Several linearly degenerate relations between these two parameters have recently been measured. Here, large scale structure correlations at $\sim 100-150 h^{-1} \mathrm{Mpc}$ are found in the comoving three-dimensional separations of redshift $z \approx 2$ quasars. These function as a comoving standard rod of length $L_{\mathrm{LSS}} \approx 130 \pm 10 h^{-1} \mathrm{Mpc}$. A local maximum in the correlation function at $\approx L_{\mathrm{LSS}} / 2$ also appears to be significant. By combining separate radial and tangential standard ruler analyses, the lifting of the $\Omega_{\mathrm{m}}-\Omega_{\Lambda}$ linear degeneracy within a single data set is demonstrated for the first time.
\end{abstract}

Key words. cosmology: observations - cosmology: theory - distance scale - quasars: general - large-scale structure of Universe - reference systems

\section{Introduction}

In standard cosmology (Weinberg 1972), space is a 3-manifold (Schwarzschild 1900; Luminet \& Roukema 1999) of nearly constant curvature, i.e. space is approximately locally homogeneous. Geometric ways of measuring average curvature include the use of phenomena of intrinsically fixed brightness or length scale, i.e. of standard candles (Perlmutter et al. 1999; Riess et al. 1998) and standard rulers [e.g. Mo et al. 1992; Broadhurst \& Jaffe 1999; Roukema \& Mamon 2000; note also the microwave background angular statistical estimates (Lange et al. 2000; Balbi et al. 2000) which can loosely speaking be thought of as "theoretical" standard rulers], but have previously been found to lead to degeneracy in the $\Omega_{\mathrm{m}}-\Omega_{\Lambda}$ plane (e.g. Lineweaver 1998). However, inhomogeneities (perturbations) in density exist and can be statistically represented by a Fourier power spectrum, and are believed to gravitationally collapse and form objects such as galaxies and clusters of galaxies. Use of a characteristic feature of this spectrum at a scale $\gg 10 h^{-1} \mathrm{Mpc}$, the size of the largest bound structures, should provide a comoving standard ruler for constraining the local geometrical parameters $\left(\Omega_{\mathrm{m}}, \Omega_{\Lambda}\right)$.

Send offprint requests to: B. F. Roukema
Many observations of both galaxies and superclusters of galaxies indicate that the maximum in the power spectrum is peaked at a wavenumber $2 \pi / L_{\mathrm{LSS}}$, where $L_{\mathrm{LSS}} \pm \Delta L_{\mathrm{LSS}} \approx 130 \pm 10 h^{-1} \mathrm{Mpc}$ (Broadhurst et al. 1990; Gaztañaga \& Baugh 1998; Einasto et al. 1997b; Deng et al. 1996) (comoving length units). Since this standard ruler should be valid independently of orientation, the different $\Omega_{\mathrm{m}}-\Omega_{\Lambda}$ degeneracies implied in the radial and tangential applications of the ruler should enable lifting of the degeneracy within a single data set, providing a potentially more powerful ruler than previous standard rulers or standard candles.

It should be noted that while the existence of a broad maximum in the power spectrum is uncontroversial, not all observational analyses agree on whether or not there is a sharp feature in the power spectrum in this region in addition to the broad maximum, and there is not yet any clear agreement on the characteristic scale of the broad maximum. For example, on one hand, Einasto et al.'s (1997b) analysis of superclusters suggests a sharp peak at $k=2 \pi / L \sim 0.05 \mathrm{~h} \mathrm{Mpc}^{-1}$. But, on the other hand, while in the low redshift IRAS PSCz (point source catalogue redshift) survey (Sutherland et al. 1999), there is, at least, a broad maximum at around $0.02 h \mathrm{Mpc}^{-1} \lesssim k=$ $2 \pi / L \lesssim 0.04 h \mathrm{Mpc}^{-1}$ (Fig. 1 of Sutherland et al. 1999), i.e. $320 h^{-1} \mathrm{Mpc} \gtrsim L \gtrsim 160 h^{-1} \mathrm{Mpc}$, there is no obvious 

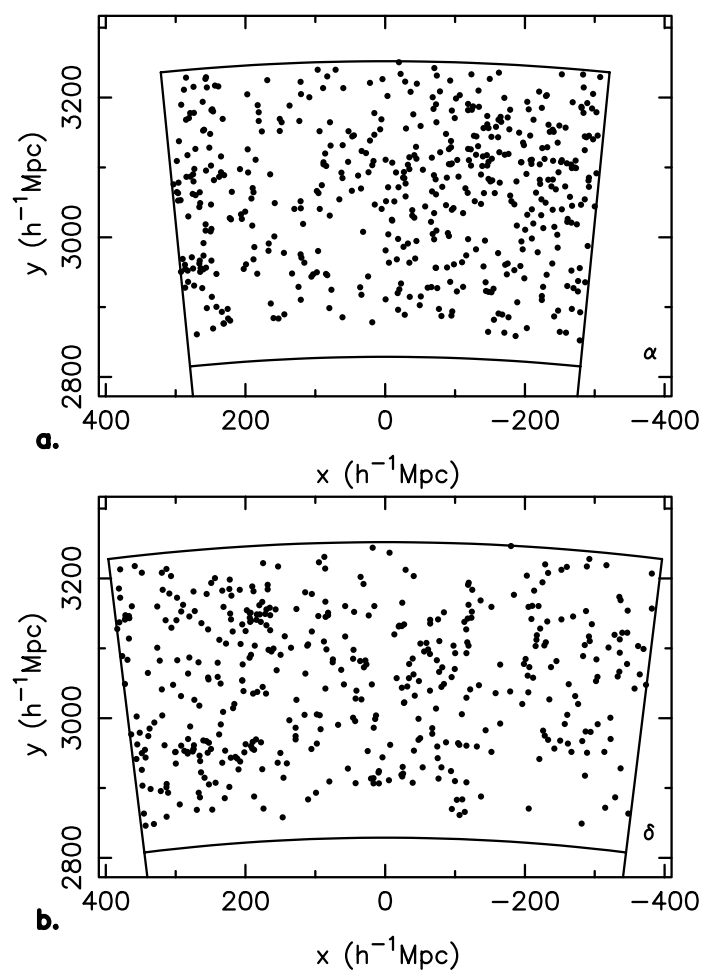

Fig. 1. Projected comoving spatial distributions of the two quasar subsamples at redshifts $1.8 \leq z<2.4$, for $\left(\Omega_{\mathrm{m}}=\right.$ $0.4, \Omega_{\lambda}=0.6$ ). a) The right ascension subsample, for $\alpha$ increasing from left to right. b) The declination subsample for $\delta$ increasing from left to right. The discrete redshifts published (Iovino et al. 1996) are converted to continuous values by uniform random offsets. The latter are used below. Differences in magnitude limits, hence different number densities, are visible in the $\alpha$ subsample and are corrected for in both subsamples (cf. Table 1). What appears to be a cluster or a supercluster in b) at $(+200,3150) h^{-1} \mathrm{Mpc}$ is located at $\left(\alpha \approx 1^{\mathrm{h}} 00^{\mathrm{m}}, \delta \approx-38.5^{\circ}, z \approx 2.24\right)$

sharp feature in this region. Nevertheless, there is a $2 \sigma$ significant sharp peak (see Fig. 1 and comment in Sect. 6 of Sutherland et al. 1999) which lies at $0.07 \mathrm{~h} \mathrm{Mpc}^{-1} \lesssim$ $k \lesssim 0.10 h \mathrm{Mpc}^{-1}$, i.e. $90 h^{-1} \mathrm{Mpc} \gtrsim L \gtrsim 60 h^{-1} \mathrm{Mpc}$ in the PSCz.

Possible reasons why the $L_{\mathrm{LSS}}=130 h^{-1} \mathrm{Mpc}$ feature found by other authors, if real, might have been missed in the Sutherland et al. (1999) analysis include

(i) use of a different population (lacking in early type galaxies);

(ii) use of too large a bin size, i.e. too much smoothing;

(iii) redshift distortion (velocity dispersion at small scales, smooth infall at larger scales), though this is discussed briefly in the paragraph preceding Sect. 3.1 of Sutherland et al. (1999);

(iv) assumption of zero cosmological constant [e.g. if $\left(\Omega_{\mathrm{m}}=0.3, \Omega_{\Lambda}=0.7\right)$, then the length scale to $c z=45000 \mathrm{~km} \mathrm{~s}^{-1}$ is underestimated by $7 \%$ ];

(v) assumption of zero curvature by use of a power spectrum analysis.
The method of Roukema \& Mamon (2000) did not assume the $L_{\text {LSS }}$ feature to be sharp, though a broad feature would obviously have given a less significant (or maybe an insignificant) signal. In the present analysis, a reasonably sharp (but low amplitude) feature consistent with $L_{\mathrm{LSS}} \sim 130 h^{-1} \mathrm{Mpc}$ is found. A secondary feature consistent with the $k \sim 0.08 h^{-1} \mathrm{Mpc}$ feature of Sutherland et al. (1999) is also found, but is not studied in detail.

Physics which could potentially be investigated in order to explain the feature at $L_{\mathrm{LSS}}$ includes acoustic oscillations in the baryon-photon fluid before last scattering, in high baryon density models (Eisenstein 1998; Meiksin et al. 1998; Peebles 1999), and features from Planck epoch physics which transfer to oscillations in the post-inflation power spectrum, for weakly coupled scalar field driven inflationary models (Martin \& Brandenberger 2000). At high redshift, the $L_{\mathrm{LSS}}=130 \pm 10 h^{-1} \mathrm{Mpc}$ feature ["distance" means comoving proper distance (Weinberg 1972) throughout this Paper] has been detected among quasars (Roukema \& Mamon 2000; Deng et al. 1994) and Lymanbreak galaxies (Broadhurst \& Jaffe 1999).

Most applications of standard candles or standard rulers exploit either the radial redshift-distance relation (Broadhurst \& Jaffe 1999) or the tangential redshiftdistance-angle relation (Perlmutter et al. 1999; Riess et al. 1998; Roukema \& Mamon 2000; Lange et al. 2000; Balbi et al. 2000), but not both simultaneously.

Alcock \& Paczyñski (1979) suggested the idea of using both constraints simultaneously, and suggested applying it at quasi-linear or non-linear scales, i.e. $r \lesssim 10 h^{-1} \mathrm{Mpc}$, but did not discuss how to lift the degeneracy in the two curvature parameters $\left(\Omega_{\mathrm{m}}, \Omega_{\Lambda}\right)$ which remains after using the local isotropy constraint, though they did suggest a theoretical method for separating out some of the peculiar velocity effects which are important at these small scales. Phillipps (1994), Matsubara \& Suto (1996), Ballinger et al. (1996) and Popowski et al. (1998) followed up this idea, demonstrating specific formulae and calculations regarding quasar pairs and the two-point autocorrelation functions of galaxies and quasars, including separation of local isotropy ("sphericity") and some of the peculiar velocity effects.

However, by using a standard ruler in the linear regime, i.e. by using a feature at $L_{\mathrm{LSS}} \approx 130 h^{-1} \mathrm{Mpc}$, peculiar velocity effects become negligible, and the inability of this scale to evolve in a Hubble time provides an additional constraint in the $\left(\Omega_{\mathrm{m}}, \Omega_{\Lambda}\right)$ plane. For the $r \lesssim 10 h^{-1} \mathrm{Mpc}$ auto-correlation function, the peculiar velocity effects are certainly important, and evolution in the length scale must be contended with, for example by model-dependent assumptions.

\section{Observational analysis and discussion}

In a previous analysis (Roukema \& Mamon 2000) of a deep, dense, homogeneous quasar survey (Iovino et al. 1996), only the tangential relation was used, to ensure that observational selection effects well known to cause 
Table 1. The two angular subsamples of the quasar candidate catalogue (Iovino et al. 1996), defined by B1950 limits $\left(\alpha_{1} \leq\right.$ $\left.\alpha \leq \alpha_{2}, \delta_{1} \leq \delta \leq \delta_{2}\right)$ and redshift limits $1.8 \leq z<2.4$. In Sect. 2.1, these are further divided into redshift subsamples $1.8 \leq z<2.1$ and $2.1 \leq z<2.4$, yielding the four independent subsamples whose analyses are shown in Figs. 2, 3 and 4. In Sect. 2.2 (Fig. 5), $1.8 \leq z<2.4$ is used, i.e. yielding just two subsamples. When generating Poisson simulations for a given subsample, further subdivisions, at $\alpha^{\prime}$ for the right ascension subsamples, and at $\delta^{\prime}, \delta^{\prime \prime}$ for the declination subsamples, are defined in order to allow for variation in magnitude zero points or magnitude cutoffs between the different plates. That is, for a given subsample with several subdivisions $i$ defined by $\alpha^{\prime}$ or $\delta^{\prime}, \delta^{\prime \prime}$, there are $n_{i}$ quasars in each subdivision $i$. For each subdivision $i, n_{i}$ uniform random $\alpha$ or $\delta$ values are generated in the appropriate $\alpha$ or $\delta$ range for that subdivision. This mimicks the magnitude limits. The subdivisions $\alpha^{\prime}, \delta^{\prime}$ and $\delta^{\prime \prime}$ are then ignored when calculating $\xi$. Numbers of objects $N$ are indicated

\begin{tabular}{lccccccc}
\hline$\alpha_{1}$ & $\alpha_{2}$ & $\delta_{1}$ & $\delta_{2}$ & $\alpha^{\prime}$ & $\delta^{\prime}$ & $\delta^{\prime \prime}$ & $N$ \\
\hline "Right ascension $(\alpha)$ subsample" & & & \\
$1^{\mathrm{h}} 00^{\mathrm{m}}$ & $1^{\mathrm{h}} 59^{\mathrm{m}}$ & -42.0 & -37.5 & $1^{\mathrm{h}} 07^{\mathrm{m}}$ & & & 500 \\
"Declination $(\delta)$ & subsample" & & & & \\
$0^{\mathrm{h}} 42^{\mathrm{m}}$ & $1^{\mathrm{h}} 00^{\mathrm{m}}$ & -42.0 & -28.0 & -37.5 & -32.5 & 453 & \\
\hline
\end{tabular}

non-cosmological periodicities in redshifts (Scott 1991) could not bias the result. In the present analysis of the high grade quasar candidate catalogue (Table 1, Fig. 1), the technique of "redshift scrambling" (see Fig. 2 caption) is used to enable use of three-dimensional information in a way that avoids redshift selection effects. Since the redshifts used in the random and observational catalogues consist of exactly the same set of numbers, any redshift selection effects, which are independent of angle, should statistically cancel out (Osmer 1981) in calculation of the correlation function $\xi(r)$ (Groth \& Peebles 1977). Since some real signal could also cancel out, in principle, this implies a conservative estimate of $\xi(r)$, i.e. a lower limit to $|\xi(r)|$.

\subsection{Local maxima in the 3-dimensional correlation function}

Figures 2 and 3 show that, for reasonable values of $\left(\Omega_{\mathrm{m}}, \Omega_{\Lambda}\right)$, a local maximum in the correlation function consistent with $L_{\mathrm{LSS}}=130 \pm 10 h^{-1} \mathrm{Mpc}$ is clearly present. By contrast, an $\left(\Omega_{\mathrm{m}}=1, \Omega_{\Lambda}=0\right)$ universe would require this local maximum to occur at $L \approx 100 h^{-1} \mathrm{Mpc}$, in contradiction with the low redshift estimates of $L_{\mathrm{LSS}}$. A correlation function consistent with the standard (Groth \& Peebles 1977) galaxy-galaxy correlation function $\xi(r) \approx$ $\left(r / 5 h^{-1} \mathrm{Mpc}\right)^{-1.8}$ is also present for $r \lesssim 40 h^{-1} \mathrm{Mpc}$.

What is the significance of the $L_{\mathrm{LSS}}$ peak? This depends on where the zero level of correlation lies. In correlation function estimates where both sample and correlation are small, the problem of only having a finite volume often requires a correction known as the integral constraint (Groth \& Peebles 1977), which most often increases the precorrected values of $\xi$. Making an integral

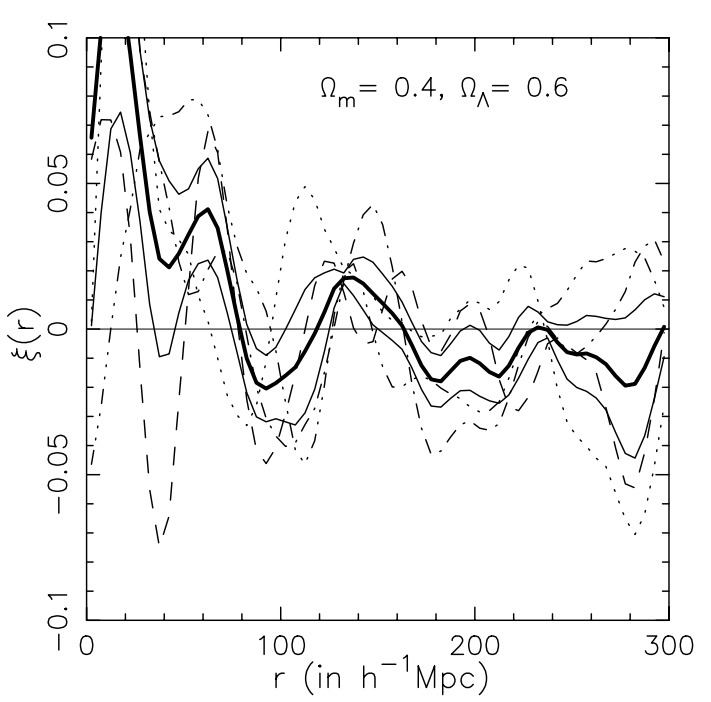

Fig. 2. Spatial two-point auto-correlation function $\xi(r)$, for separations $r$ in comoving units and $\left(\Omega_{\mathrm{m}}=0.4, \Omega_{\Lambda}=0.6\right)$. The four angular/redshift subsamples are shown as dashed $(\delta, 1.8 \leq z<2.1)$, dashed-dotted $(\delta, 2.1 \leq z<2.4)$, dotted $(\alpha, 1.8 \leq z<2.1)$ and dashed-triple-dotted $(\alpha, 2.1 \leq z<2.4)$ curves. The mean $\langle\xi\rangle$ and the standard error in the mean $\sigma_{\langle\xi\rangle}$ are shown by the thick and thin solid lines respectively. The correlation functions are calculated in three-dimensional curved space via $\xi(r)=\left(D D-2 D R / n+R R / n^{2}\right) /\left(R R / n^{2}\right)$ where $D D, D R$ and $R R$ indicate numbers of data-data, data-random and random-random quasar pairs respectively (Landy \& Szalay 1993), and $n=20$ times more random points than data points are used. The random catalogues use (i) uniform probability distributions in the two angular directions (Table 1), and (ii) random permutations (" $z$ scrambles", IIIb in Osmer 1981) of the observational set of redshifts, to avoid biases from redshift selection effects (Scott 1991). Bin size is $5 h^{-1} \mathrm{Mpc}$ and $\xi$ is smoothed by a Gaussian with $\sigma=10 h^{-1} \mathrm{Mpc}$. The low values of $\xi$ at $r \lesssim 20 h^{-1} \mathrm{Mpc}$ are related to redshift roundoff error

constraint correction usually requires assumptions on the intrinsic shape of $\xi$. To avoid these assumptions, it is more prudent just to quantify the peak as a local maximum (Deng et al. 1994; Roukema \& Mamon 2000). For a maximum at $r_{\max }$ consistent with a peak at $f L_{\mathrm{LSS}}$, where $f=1$, define $\xi^{r}, \xi_{-}$and $\xi_{+}$as the maximum value and the first minima below and above $r_{\max }$ respectively, and take the maximum value of $\sigma_{\langle\xi\rangle}(r)$ for $r \in\left[r_{\max }-\right.$ $\left.f \Delta L_{\mathrm{LSS}}, r_{\mathrm{max}}+f \Delta L_{\mathrm{LSS}}\right]$, where $\Delta L_{\mathrm{LSS}}=10 h^{-1} \mathrm{Mpc}$. Then,

$(S / N)_{f} \equiv \frac{\xi^{r}-\left(\xi_{-}+\xi_{+}\right) / 2}{\max \left\{\sigma_{\langle\xi\rangle}(r)\right\}}$.

yields $(S / N)_{1}=3.2$ for $\left(\Omega_{\mathrm{m}}, \Omega_{\Lambda}\right)=(0.4,0.6)$.

Figure 4 shows that an automatic search for this peak, using a simple and robust method, i.e. using the value of $r$ for which $\xi(r)$ is maximum over a very large interval in $r$, yields an approximately linear confidence band in the $\left(\Omega_{\mathrm{m}}, \Omega_{\Lambda}\right)$ plane. Since this band is consistent with kinematical (Carlberg et al. 1997; Mamon 1993) and baryonic fraction (White et al. 1993; Henriksen \& Mamon 1994) 

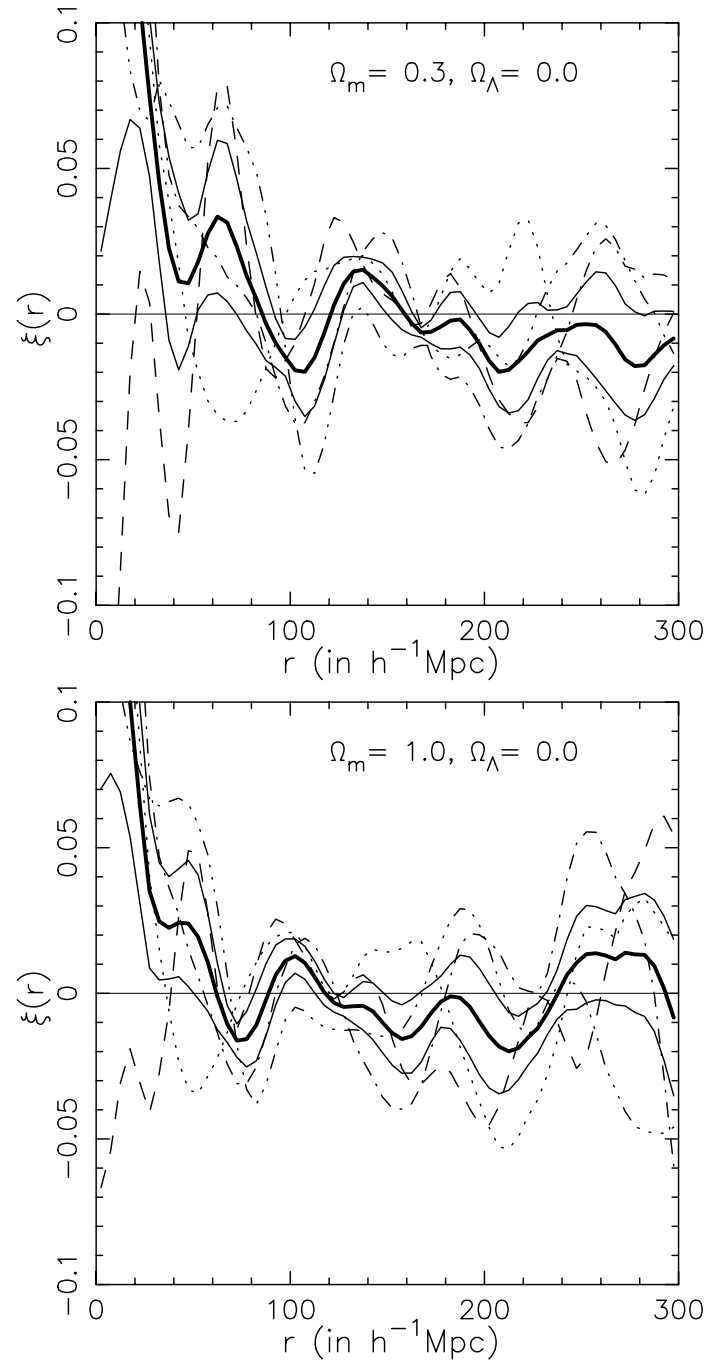

Fig. 3. Correlation function $\xi(r)$, as for Fig. 2, but for a hyperbolic universe $\left(\Omega_{\mathrm{m}}=0.3, \Omega_{\Lambda}=0.0\right)$ above, and a zero cosmological constant, flat universe $\left(\Omega_{\mathrm{m}}=1.0, \Omega_{\Lambda}=0.0\right)$ below

Table 2. Probability (Eq. 2) of finding one, two or three peaks among 200 random simulations at $f L_{\mathrm{LSS}}$ where $f=0.5,1$ and/or 2 , for $\left(\Omega_{\mathrm{m}}, \Omega_{\Lambda}\right)=(0.4,0.6)$

\begin{tabular}{ccccccc}
\hline$P_{0.5}^{\text {sim }}$ & $P_{1}^{\text {sim }}$ & $P_{2}^{\text {sim }}$ & $P_{0.5,1}^{\text {sim }}$ & $P_{1,2}^{\text {sim }}$ & $P_{0.5,2}^{\text {sim }}$ & $P_{0.5,1,2}^{\text {sim }}$ \\
0.025 & 0.01 & 0.335 & $<0.005$ & 0.005 & 0.005 & $<0.005$ \\
\hline
\end{tabular}

constraints for clusters and groups of galaxies, though to slightly higher $\Omega_{\mathrm{m}}$ values than were found in the purely tangential analysis of the present survey (Roukema \& Mamon 2000), the coincidence would be surprising if it were due to noise or systematic effects.

Moreover, what appear to be peaks at $L_{\mathrm{LSS}} / 2 \pm$ $\Delta L_{\mathrm{LSS}} / 2$ and near $2 L_{\mathrm{LSS}} \pm 2 \Delta L_{\mathrm{LSS}}$ are present, though to lower significance, with $(S / N)_{0.5}=2.3$ and $(S / N)_{2}=1.2$ respectively, for $\left(\Omega_{\mathrm{m}}, \Omega_{\Lambda}\right)=(0.4,0.6)$. Could any of the three peaks be induced by noise which has common statistical properties among all the four subsets, either due to shot noise or selection effects? Redshift selection effects

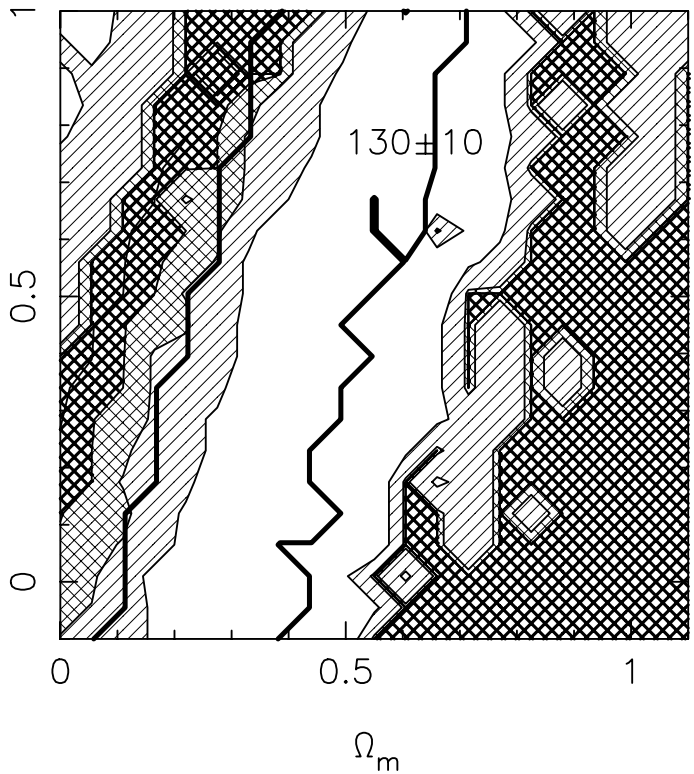

Fig. 4. Confidence intervals for rejecting the presence of a peak at $L_{\mathrm{LSS}} \pm \Delta L_{\mathrm{LSS}}$ for various hypotheses on $\left(\Omega_{\mathrm{m}}, \Omega_{\Lambda}\right)$. Rejection levels are $1-P>0 \%$ (white), $1-P>68 \%$ (hatched), $1-P>95 \%$ (light cross-hatched) and $1-P>99.7 \%$ (heavy cross-hatched). The $1-P=68 \%$ contour for testing a peak at $L_{\mathrm{LSS}} / 2 \pm \Delta L_{\mathrm{LSS}} / 2$ test is shown in bold. For each pair $\left(\Omega_{\mathrm{m}}, \Omega_{\Lambda}\right)$, the peak position is estimated as the value $r_{\max }$ for which $\xi(r)$ is maximum in $100 \leq r \leq 300 h^{-1} \mathrm{Mpc}$. (For the $L_{\mathrm{LSS}} / 2$ peak, $40 \leq r \leq 100 h^{-1} \mathrm{Mpc}$ is searched). The measurement uncertainty in the $f L_{\mathrm{LSS}}$ peak is estimated as $\Delta r_{\max }=f \Delta L_{\mathrm{LSS}}$ (for $f=0.5,1$ ). The probability of finding $r_{\max }$ close to $f L_{\mathrm{LSS}}$ assumes Gaussian errors, i.e. $P_{\mathrm{f}} \equiv$ $\operatorname{erfc}\left[\left|r_{\max }-f L_{\mathrm{LSS}}\right| /(\sqrt{2} \sigma)\right]$, where $\sigma^{2}=\left(\Delta r_{\max }\right)^{2}+\left(f \Delta L_{\mathrm{LSS}}\right)^{2}$

have been removed by the use of $z$-scrambling. Angular selection effects may be present at a small level (see Sect. 3.4 of Roukema \& Mamon 2000), but are more likely to decrease the amplitude of any signal rather than introduce false correlations which mimic the signal found. Moreover, the convergence of the separate tangential and radial analyses below (Sect. 2.2) suggest that the effects of angular selection are weak.

To test the properties of shot noise, random simulations were performed as before but substituted for the data. The probabilities that maxima can occur as close to and of at least the same signal-to-noise ratio as the observed values can be defined

$$
\begin{aligned}
P_{f}^{\operatorname{sim}} \equiv & P\left[\left|r_{\mathrm{sim}}-f L_{\mathrm{LSS}}\right| \leq\left|r_{\mathrm{obs}}-f L_{\mathrm{LSS}}\right| \quad\right. \text { and } \\
& \left.(S / N)_{f}^{\mathrm{sim}} \geq(S / N)_{f}^{\mathrm{obs}}\right],
\end{aligned}
$$

where "sim" and "obs" indicate simulations and observations respectively, and the intervals in $r$ are as above. Since one might suspect a single noise feature to cause, say, simultaneous features at two or three of the positions, mimicking the observed signal, the probabilities of finding the peaks might not be independent, e.g. $P_{f_{1}, f_{2}}^{\mathrm{sim}} \approx P_{f_{1}}^{\mathrm{sim}} \approx$ $P_{f_{2}}^{\operatorname{sim}} \gg P_{f_{1}}^{\operatorname{sim}} P_{f_{2}}^{\text {sim }}$ for $f_{1}, f_{2} \in\{0.5,1,2\}$ is in principle possible. The results (Table 2) show that the hypotheses of 

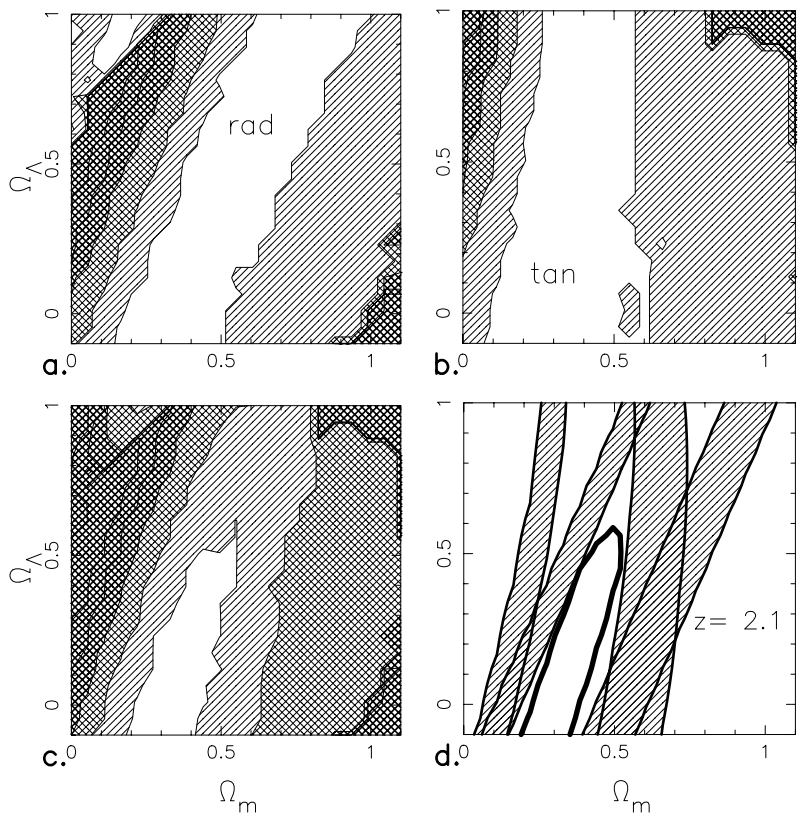

Fig. 5. Partial lifting of the $\Omega_{\mathrm{m}}-\Omega_{\Lambda}$ degeneracy. Confidence intervals are as for Fig. 4 for a) the radial $\left(1-P_{1}^{\text {rad }}\right)$, b) tangential $\left(1-P_{1}^{\mathrm{tan}}\right)$ and c) combined $\left(1-P_{1}^{\mathrm{rad}} P_{1}^{\mathrm{tan}}\right)$ constraints. d) A simple model, for which the $95 \%>1-P>68 \%$ contours for radial (straight contours) and tangential (curved, nearly vertical contours) constraints showing near linear degeneracy are shaded as before (hatched regions); and the combined $68 \%>1-P>0 \%$ contour is the half-ellipse-like shape nested between these (heavy contour). a-c) The radial and tangential tests are performed for the $L_{\mathrm{LSS}} \pm \Delta L_{\mathrm{LSS}}$ peak as in Fig. 4 , except that only pairs oriented within $30^{\circ}$ of the radial and tangential directions (respectively) are included in calculation of $\xi(r)$. Both angular subsamples over $1.8 \leq z<2.4$ are used. The radial and tangential tests are assumed to be statistically independent. d) For the model, the redshift interval $\Delta z$ and the angle $\theta$ corresponding to $L_{\mathrm{LSS}}$, assuming that $\left(\Omega_{\mathrm{m}}=0.3, \Omega_{\Lambda}=0.0\right)$ and that $z=2.1$, are calculated. For each pair $\left(\Omega_{\mathrm{m}}, \Omega_{\Lambda}\right)$, the radial and tangential distance intervals implied by $\Delta z$ and $\theta$ are calculated, ignoring the initial assumption about $\Omega_{\mathrm{m}}$ and $\Omega_{\Lambda}$. These are treated as two independent "experiments", and Gaussian probabilities $P^{\mathrm{rad}}, P^{\mathrm{tan}}$ of observing these values, given $L_{\mathrm{LSS}}, \Delta L_{\mathrm{LSS}}, \Omega_{\mathrm{m}}$ and $\Omega_{\Lambda}$ are calculated as before. The combined rejection is $1-P^{\text {rad }} P^{\text {tan }}$

either of the $L_{\mathrm{LSS}} / 2$ or the $L_{\mathrm{LSS}}$ peaks occurring by chance are each rejected to $1-P^{\operatorname{sim}}>97 \%$, and that of both occurring simultaneously is rejected to $1-P^{\operatorname{sim}}>99.5 \%$. The hypothesis of the the $2 L_{\mathrm{LSS}}$ peak occurring by shot noise cannot be significantly rejected. These values vary throughout the $\left(\Omega_{\mathrm{m}}, \Omega_{\Lambda}\right)$ plane.

Figure 4 shows that the $68 \%>1-P>0 \%$ confidence intervals for the $L_{\mathrm{LSS}} / 2$ and $L_{\mathrm{LSS}}$ peaks are consistent.

\subsection{Tangential versus radial correlations}

A standard ruler should not depend on orientation. Can use of both radial and tangential information lift the degeneracy of $\left(\Omega_{\mathrm{m}}, \Omega_{\Lambda}\right)$ constraints? To illustrate this, the full redshift interval $1.8 \leq z<2.4$ is used, but only pairs of objects within $30^{\circ}$ of either the radial or tangential directions respectively are used. For the geometry of this survey, about $10 \%$ of pairs are radial and $60 \%$ of pairs are tangential according to this criterion.

Figure 5 clearly shows, both observationally and theoretically, the difference in the slopes of the radial and tangential constraints at $z \approx 2$. A hyperbolic $\left(\Omega_{\mathrm{m}}+\Omega_{\Lambda}-1<\right.$ $0)$ universe is suggested by the $68 \%$ confidence limit, though a flat universe with $\Omega_{\Lambda}=1-\Omega_{\mathrm{m}}=0.5$ is only rejected to $1-P \approx 80 \%$ confidence, i.e. not significantly. The partially lifted degeneracy can be represented (at $68 \%$ confidence) as

$\Omega_{\mathrm{m}}=(0.30 \pm 0.04) \Omega_{\Lambda}+(0.30 \pm 0.11), \quad \Omega_{\Lambda}<0.5$.

This suggests a somewhat higher matter density and lower cosmological constant than other recent results.

\section{Conclusion}

The confirmation of the $L_{\mathrm{LSS}}$ peak and the partial lifting of the $\Omega_{\mathrm{m}}-\Omega_{\Lambda}$ degeneracy show that ongoing and future large quasar surveys [in particular the 2 Degree Field Quasar Survey (Boyle et al. 2000) and the Sloan Digital Sky Survey quasar sample (e.g. Fan et al. 2000)] will have a much more powerful tool for local geometrical constraints than was previously thought. While local isotropy of the $r \lesssim 10 h^{-1} \mathrm{Mpc}$ scale correlation function can in principle be used as a local geometrical constraint, a standard ruler at $L_{\mathrm{LSS}} \approx 130 h^{-1} \mathrm{Mpc}$ has the advantages (i) of being little affected by peculiar velocities, and (ii) of occurring well into the linear regime where evolution within a Hubble time is unlikely.

Moreover, the detection of the $L_{\mathrm{LSS}} / 2$ peak (cf. Fig. 6 of Tadros \& Efstathiou 1996; Fig. 1 of Sutherland et al. 1999; Fig. 3 of Mo et al. 1992) implies that both peaks might either be signs of high baryon density (Eisenstein 1998; Meiksin et al. 1998; Peebles 1999) or of preinflationary physics (Martin \& Brandenberger 2000), enabling constraints to be put on these. For increased confidence in this method, more precise low redshift constraints on large scale structure features near $L_{\mathrm{LSS}} \approx 130 h^{-1} \mathrm{Mpc}$ will be highly desirable. Results from the 2 Degree Field Galaxy Redshift Survey (e.g. Folkes et al. 1999), the Sloan Digital Sky Survey galaxy sample (York et al. 2000), and the 6 Degree Field galaxy survey (e.g. Mamon 1998) may help for these low redshift calibrations.

Acknowledgements. We thank Emmanuel Bertin, Stéphane Colombi, Georges Maignan, S. Sridhar and the referee, Pat Osmer, for useful comments. B.F.R. thanks the Institut d'Astrophysique de Paris, CNRS and DARC, Observatoire de Paris, for their hospitality, and acknowledges the support of la Société de Secours des Amis des Sciences. Data are available at http://cdsweb.u-strasbg.fr/cgi-bin/Cat?J/A+AS/119/265. 


\section{References}

Alcock, C., \& Paczyñski, B. 1979, Nat, 281, 358

Balbi, A., et al. 2000, in press, ApJL [arXiv: astro-ph/0005124]

Ballinger, W. E., Peacock, J. A., \& Heavens, A. F. 1996, MNRAS, 282, 877

Boyle, B. J., Shanks, T., Croom, S. M., et al. 2000, MNRAS, 317, 1014 [arXiv:astro-ph/0005368]

Broadhurst, T. J., Ellis, R. S., Koo, D. C., \& Szalay, A. S. 1990, Nature, 343, 726

Broadhurst, T., \& Jaffe, A. H. 2000, in press, ApJL [arXiv: astro-ph/9904348]

Carlberg, R. G., Yee, H. K. C., \& Ellingson, E. 1997, ApJ, 478, 462

Deng, X.-F., Deng, Z.-G., \& Xia, X.-Y. 1996, Chin. Astron. Astroph., 20, 383

Deng, Z.-G., Xia, X.-Y., \& Fang, L.-Zh. 1994, ApJ, 431, 506

Einasto, J., et al. 1997, Nature, 385, 139

Eisenstein, D. J., Hu, W., Silk, J., \& Szalay, A. S. 1998, ApJ, 494, L1

Fan, X., et al. 2000, AJ, 120, 1167

Folkes, S., et al. 1999, MNRAS, 308, 459

Gaztañaga, E., \& Baugh, C. M. 1998, MNRAS, 294, 229

Groth, E. J., \& Peebles, P. J. E. 1977, ApJ, 217, 385

Henriksen, M. J., \& Mamon, G. A. 1994, ApJ, 421, L63

Iovino, A., Clowes, R., \& Shaver, P. 1996, A\&AS, 119, 265

Landy, S. D., \& Szalay, A. S. 1993, ApJ, 412, 64

Lange, A. E., et al. 2000 [arXiv:astro-ph/0005004]

Lineweaver, C. H. 1998, ApJ, 505, L69

Luminet, J.-P., \& Roukema, B. F. 1999, in Theoretical and Observational Cosmology, ed. M. Lachièze-Rey (Kluwer, Dordrecht), 117 [arXiv:astro-ph/9901364]
Mamon, G. A. 1993, in The N-Body Problem \& Gravitational Dynamics, ed. F. Combes, \& E. Athanassoula (Obs. de Paris, Paris), 188 [arXiv:astro-ph/9308032]

Mamon, G. A. 1998, in XIVth IAP Mtg "Wide-Field Surveys in Cosmology", ed. S. Colombi, Y. Mellier, \& B. Raban (Paris: Éditions Frontières), 323 [arXiv:astro-ph/9809376]

Martin, J., \& Brandenberger, R. H. 2000 [arXiv: astro-ph/0005432]

Matsubara, T., \& Suto, Y. 1996, ApJ, 470, L1

Meiksin, A., White, M., \& Peacock, J. A. 1999, MNRAS, 304, 851 [arXiv: astro-ph/9812214]

Mo, H. J., Xia, X.-Y., Deng, Z.-G., Börner, G., \& Fang, L. Z. 1992, A\&A, 256, L23

Osmer, P. S. 1981, ApJ, 247, 762

Peebles, P. J. E. 1999, ApJ, 510, 531

Perlmutter, S., et al. 1999, ApJ, 517, 565 [arXiv: astro-ph/9812133]

Phillipps, S. 1994, MNRAS, 269, 1077

Popowski, P. A., Weinberg, D. H., Ryden, B. S., \& Osmer, P. S. 1998, ApJ, 498, 11

Riess, A. G., et al. 1998, AJ, 116, 1009

Roukema, B. F., \& Mamon, G. A. 2000, A\&A, 358, 395 [arXiv: astro-ph/9911413]

Schwarzschild, K. 1900, Vier. d. Astr. Gess., 35, 337

Scott, D. 1991, A\&A, 242, 1

Sutherland, W., et al. 1999, MNRAS, 308, 289

Tadros, H., \& Efstathiou, G. 1996, MNRAS, 282, 1381

White, S. D. M., Navarro, J. S., Evrard, A. E., \& Frenk, C. S. 1993, Nature, 366, 429

Weinberg, S. 1972, Gravitation and Cosmology: Principles and Applications of the General Theory of Relativity (Wiley, New York)

York, D. G., et al. 2000, AJ, 120, 1579 